\title{
Full-Wave Analysis of Power Distribution Networks in Printed Circuit Boards
}

\author{
Francescaromana MARADEI $^{\dagger \mathrm{a})}$, Spartaco CANIGGIA ${ }^{\dagger \dagger \mathrm{b})}$, Nicola INVERARDI ${ }^{\dagger \dagger \dagger \mathrm{c})}$, \\ and Mario ROTIGNI ${ }^{\dagger \dagger \dagger}$, Nonmembers
}

\begin{abstract}
SUMMARY This paper provides an investigation of power distribution network (PDN) performance by a full-wave prediction tool and by experimental measurements. A set of six real boards characterized by increasing complexity is considered in order to establish a solid base for behaviour understanding of printed circuit boards. How the growing complexity impacts on the board performance is investigated by measurements and by simulations. Strengths and weakness of PDN modeling by the full-wave software tool Microwave Studio are highlighted and discussed.

key words: decoupling capacitor, integrated circuits (ICs), power distribution network $(P D N)$, printed circuit board $(P C B)$, radiated and conducted emission, SPICE circuit simulator
\end{abstract}

\section{Introduction}

The performance required by modern microcontrollers in automotive applications is becoming more and more demanding in terms of integration, execution speed and reliability. The IEC61967 standard [1] recommends to perform radiated emission test using a test board, sized to fit the TEM cell aperture, and exposing the device under test (DUT) to the detector structure inside the cell. The use of a suitable board is of major importance to minimize its contribution to the measured emissions. For this reason, great attention must be addressed to the design of the power distribution network (PDN). A proper understanding and characterization of PDN are very important to avoid or at least clearly identify emission peaks due to resonances on the PCBs and/or coming from discontinuities in the impedance profile versus frequency. The confidence acquired in the board performance allows one to better focus on real DUT emissions, screening out from the debugging process the parasitic contribution of the test environment. The main purpose of the board-level PDN is to distribute stable power from the voltage module regulator (VMR) to the electronics. However,

\footnotetext{
Manuscript received March 2, 2010.

Manuscript revised March 5, 2010.

${ }^{\dagger}$ The author is with the Sapienza University, Department of Electrical Engineering, Via Eudossiana 18, 00184 Rome, Italy.

${ }^{\dagger}$ The author is an EMC Consultant, Viale Moranti 7, 20010 Bareggio (MI), Italy.

${ }^{\dagger \dagger}$ The author is with EURO Instruments, Via dell'Industria 29/31, 25032 Chiari (BS), Italy.

${ }^{\dagger \dagger \dagger}$ The author is with STMicroelectronics, Via C. Olivetti, 20041 Agrate Brianza, Italy.

a)E-mail: fr.maradei@ieee.org

b) E-mail: spartaco.caniggia@ieee.org

c) E-mail: nicola.inverardi@euroinstruments.it

d) E-mail: mario.rotigni@st.com

DOI: $10.1587 /$ transcom.E93.B.1670
}

switching circuitry demands static and dynamic current, which across the PDN impedance $Z_{\mathrm{PDN}}$ causes the voltage to fluctuate at the chip's power-supply terminals [2]. The noise circulating into power and ground planes can affect the operation of other active devices (signal/power integrity), as well as producing radiation (EMI). In order to effectively deliver power to the chip with minimal noise while reducing radiated emissions, the PDN input impedance $Z_{\text {PDN }}$ needs to be as low as possible in the frequency range of interest, from DC to several hundreds of $\mathrm{MHz}$. The use of decoupling capacitors allows to lower $Z_{\mathrm{PDN}}$ below a few hundreds of $\mathrm{MHz}$ [3], [4]. Above this frequency, their action as capacitance is affected by the parasitic inductance associated to the component itself and by the inductance associated to the component leads. In this task, simple and reliable simulation tools for the prediction of PDN resonances and of filtering performance by decoupling capacitors offer an attractive option, allowing board investigation before spending money and time in actual building a prototype. A further nice advantage is the possibility to probe the impedance at every location of the PCB, an operation that is practically impossible to do on the real board because of the probing constraints posed by instrumentation such as a network analyser. In the literature there are three main approaches used for the analysis of PDN. The first approach is based on the cavity mode model [5], [6] and allows very fast prediction of resonances for boards with simple structure. In case of complex structures the cavity model is combined to the segmentation method and its implementation requires experienced engineers. The second approach is based on PDN modeling by a SPICE circuit [7], [8]. This modeling is very convenient allowing simple, accurate and fast simulations, but it is difficult to use with complex boards having many planes and cuts. The third approach is based on the full-wave analysis by numerical methods [9]-[11]. These methods allow detailed modeling of complex configurations, but are very expensive in terms of computing time and memory requirements. The goal of this paper is to establish a solid base for PCB behaviour understanding through the investigation of six test boards which have been designed and manufactured at EURO Instruments. The test boards implement a PDN of growing complexity, from a simple pair of supply/ground planes with some decoupling capacitors, to a more complex structure with supply island, cuts in the planes, and multiple layers. The boards are modelled by the full-wave software tool MicroWave Studio (MWS), and the critical aspects to 
assure analysis effectiveness and accuracy are discussed.

\section{2. $Z_{\text {PDN }}$ versus Scattering Parameters}

The analytical relation between $\mathrm{Z}$ and $\mathrm{S}$ parameters can be found in [12]. In practical cases, except possibly at parallel resonance frequency, the approximate relation:

$$
Z_{\mathrm{PDN}} \approx 25 S_{21}
$$

can be used, since $Z_{\mathrm{PDN}}$ is much lower than the nominal characteristic impedance of the measurement system (i.e., $Z_{0}=50 \mathrm{Ohm}$ ). This equation is valid with an error less of $1 \%$ up to the frequencies near the first series resonant frequency of the PCB caused by the inductance associated with the connection of the test ports and the board capacitance [2], [4]. Ideally, in point 1 of a PCB where the IC switches, $S_{11}$ should be maximized in order to have a very low $Z_{11}$ (i.e., $Z_{\mathrm{PDN}}$ ) in the frequency range of interest. On the contrary, $S_{21}$ between point1 and point 2 where another IC is placed, should be minimized in order to have strong attenuation of the Delta-I noise generated at point1 and propagating along the PCB. Owing to the approximate expression (1), and the perfect correspondence of resonant frequencies, in the following the results will be shown in terms of scattering parameters which are directly measured by a network analyzer and calculated in a straightforward way. For brevity, the results of the $S_{21}$ parameter only are shown and commented. In any case, the discussion can be considered exhaustive since the two scattering parameters are characterized by the same resonant points.

\section{Test Board Description}

Six different test boards with increasing complexity are considered in order to allow a better understanding of the board behavior, and to highlight the scattering parameters change. For all six PCBs, the board dimensions are $10 \mathrm{~cm} \times 10 \mathrm{~cm}$, the distance between two nearby layers is $0.12 \mathrm{~mm}$, and the dielectric substrate has relative permittivity $\epsilon_{r}=4.3$ and loss $\operatorname{tangent} \tan \delta=0.02$. Some miniature coaxial connectors (SMA) are assembled on each test board in the same position to properly connect the network analyzer (NA) and allow reliable measurements. The six boards have in the same position, vias and cuts to allocate seven decoupling capacitors (indicated in the following as decaps for brevity). The nominal values of the decoupling capacitors are shown in Table 1. The description of the boards is as follows (see Figs. 1, 2) [15]:

- Board 1 has two layers: a top solid ground plane (see Fig. 1(a)), and a bottom solid power plane (as in Fig. 2(a), without the cut, the split)

- Board 2 has two layers: a top solid ground plane (see Fig. 1(a)), a bottom solid power and ground planes separated by a split (as in Fig. 2(a) without the cut)

- Board 3 is similar to Board 2, with a cut in the power plane (see Figs. 1(a) and 2(a)).
Table 1 Equivalent circuit parameters of decoupling capacitors.

\begin{tabular}{|l|c|c|c|}
\hline & $\mathrm{C}$ & ESL & ESR \\
\hline C1 & $33 \mathrm{nF}$ & $1.94 \mathrm{nH}$ & $0.297 \Omega$ \\
\hline C2 & $33 \mathrm{nF}$ & $1.94 \mathrm{nH}$ & $0.297 \Omega$ \\
\hline C3 & $100 \mathrm{nF}$ & $1.99 \mathrm{nH}$ & $0.128 \Omega$ \\
\hline C4 & $100 \mathrm{nF}$ & $1.99 \mathrm{nH}$ & $0.128 \Omega$ \\
\hline C5 & $100 \mathrm{nF}$ & $1.99 \mathrm{nH}$ & $0.128 \Omega$ \\
\hline C6 & $100 \mu \mathrm{F}$ & $2.5 \mathrm{nH}$ & $0.066 \Omega$ \\
\hline C7 & $100 \mu \mathrm{F}$ & $2.5 \mathrm{nH}$ & $0.066 \Omega$ \\
\hline
\end{tabular}

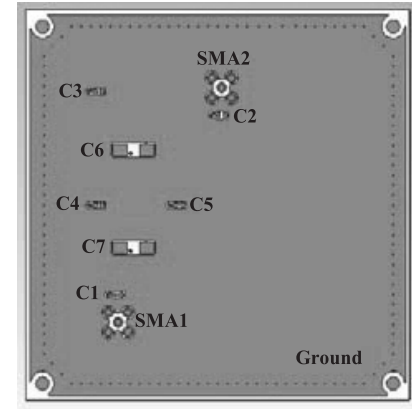

(a)

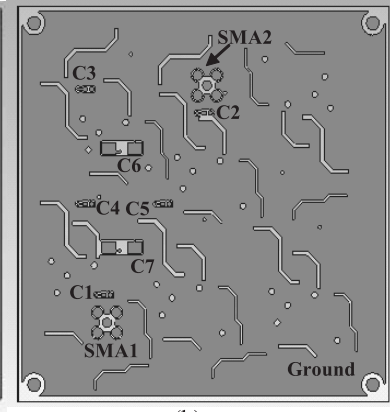

(b)
Fig. 1 Top view (ground plane) of Boards 1-5 (a), and of Board 6 (b).

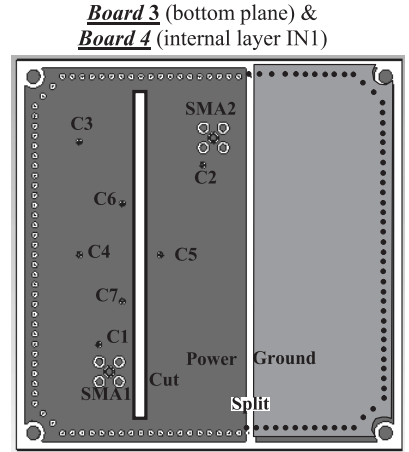

(a)

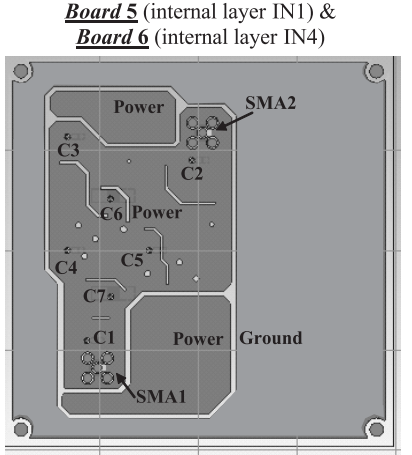

(b)
Fig. 2 Power plane view of Boards 3 and 4 (a), and 5 and 6 (b).

- Board 4 has three layers: a top and bottom solid ground planes as Fig. 1(a), and an internal IN1 solid power plane with a cut and ground plane as in Fig. 2(a).

- Board 5 has three layers: a top and bottom solid ground planes as in Fig. 1(a), an internal layer IN1 which has three islands of power (partitioning), and a ground that surrounds the power islands as in Fig. 2(b).

- Board 6 has six layers: the fourth internal layer IN4 has the same configuration of the internal layer of Board 5 (see Fig. 2(b)), while the top and bottom layers as well as the internal layers IN1, IN2 and IN3 are dedicated to ground. Top and IN1 layers have some discontinuities simulating holes and routings (see Fig. 1(b)).

The top ground plane of Boards 1-5 is shown in Fig. 1(a), while that of Board 6 is shown in Fig. 1(b). The power plane view of boards 3 and 6 is shown in Fig. 2. Note that even in Board 1 that is the simplest among those considered, there are several vias and cuts for SMA connectors and placement 
of decoupling capacitors. The usage of three layer boards is acceptable for investigation purposes even if never applied in practice due to low mechanical performance.

\section{Experimental Measurements}

An experimental investigation on the six test boards has been performed at STMicroelectronics laboratory in Cornaredo (Italy). The experimental setup is shown in Fig. 3 where a board isolated from the ground is connected to the network analyzer. The scattering parameters were measured by an Agilent E8358 network analyzer, in the frequency range $300 \mathrm{kHz}-2 \mathrm{GHz}$ and adopting 1601 points. Two port SOLT calibration were initially performed using a Hewlett Packard 85052D calibration kit. The coaxial cables used between the network analyzer and the PCB under tests equipped with SMA connectors were the armoured workhorsetype with the following characteristics: $50 \Omega$ characteristic impedance and $-100 \mathrm{~dB}$ minimum relative shielding.

The $S_{21}$ parameter has been measured for the six test boards without the presence of decoupling capacitors. The measurements of Fig. 4 show how $S_{21}$ changes as the complexity of the PCB increases. Note that for all the considered boards the same reference points 1 an 2 were adopted, being the SMA connectors located in the same position. The results for $S_{21}$ are compared using as a reference line $-20 \mathrm{~dB}$ on the graphs and the following comments can be drawn:

- Board 1 has four points of resonance due to the fact that the two parallel planes behave like a resonant cavity.

- Board 2 has higher $S_{21}$ at frequencies over $1 \mathrm{GHz}$ than Board 1 due to the fact that the area of the power plane is reduced and the ground plane is extended in the same layer of the power and separated by a narrow split (partitioning effect).

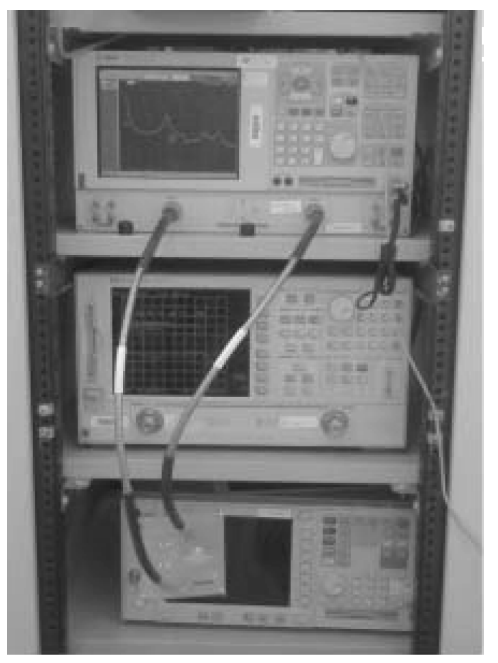

(a)

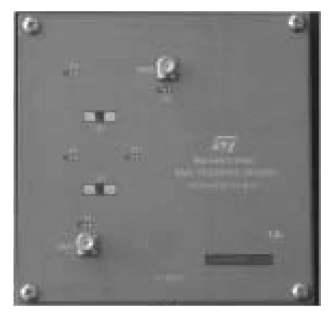

(b)

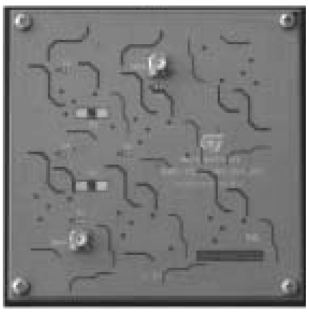

(c)
Fig. 3 Experimental activity at STMicroelectronics (Cornaredo, Italy): measurement setup (a), Board 3 (b) and Board 6 (c).
- Boards 3 and 4 have the same $S_{21}$ with points of resonance shifted towards lower frequencies compared with Board 2. This is due to the fact that board 3 has the same partitioning effect of Board 2 with in addition a long cut between point 1 and 2 .

- Board 4 is similar to Boards 3 with an additional ground plane that does not change significantly $\mathrm{S}$ parameter curve.

- Board 5 is characterized by a first point of resonance shifted towards higher frequencies and higher values of $S_{21}$ up to $2 \mathrm{GHz}$. This is due to the fact that the PCB is similar as Board 4 with the difference that partitioning effect is more complex and the area of power where the points 1 and 2 are placed is reduced.

- Board 6 has $S_{21}$ values similar to Board 5 with a first point of resonance shifted towards higher frequencies. This is a 6-layers PCB similar to real PCBs with complex partitioning and several short cuts simulating component placements and routing.

Filtering of decoupling capacitors was also investigated. The $S_{21}$ parameter in the absence and with decaps are shown in Fig. 5 in case of Boards 1, 3, and 6. The comparison shows that decoupling capacitors are ineffective in lowering $S_{21}$ parameter over $150 \mathrm{MHz}$, owing to the inductive effect associated to the capacitors. A new resonance peak is introduced at $200 \mathrm{MHz}$ by the capacitors. This is a parallel resonance due to the parasitic inductance of decaps that resonates with the PCB capacitance. Over $250 \mathrm{MHz}$ the $S_{21}$ parameters presents resonant points which depend on the structure of the PCB only, and not on decoupling capacitors that behave as a high inductive impedance. In conclusion, this investigation has shown:

- How resonance frequencies change depending on the number of layers and type of discontinuity such as vias, split, power islands, and short cuts.

- That the values of the $S_{21}$ increases at higher frequen-

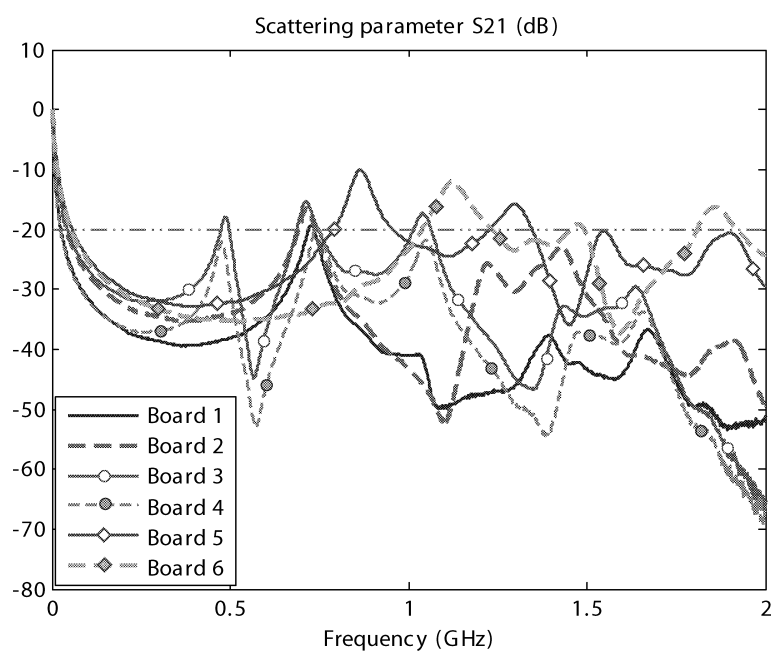

Fig. 4 Measured $S_{21}$ for the six test boards considered in the absence of decoupling capacitors. 


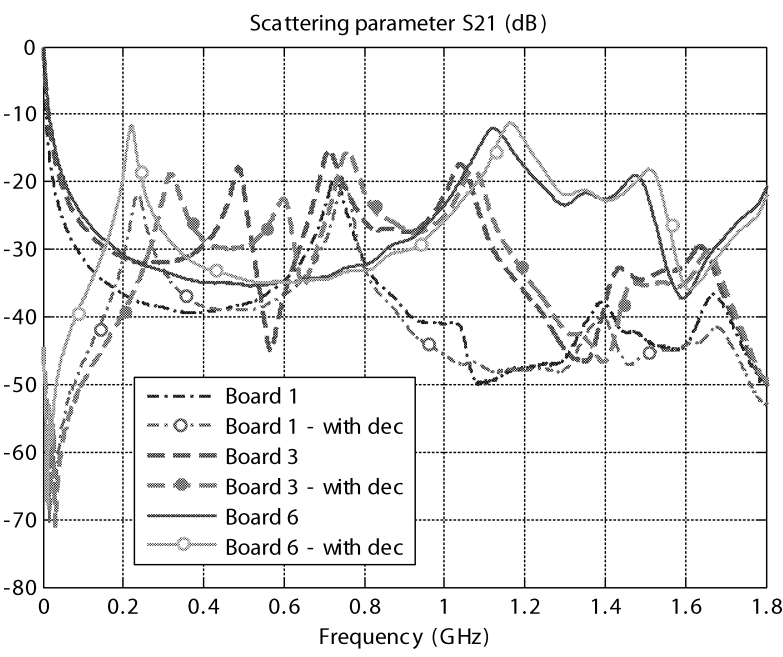

Fig.5 Measured $S_{21}$ for Boards 1, 3 and 6 in the absence and with decoupling capacitors.

cies as the board complexity.

\section{PDN Investigation by a Full-Wave Software Tool}

Full-wave numerical simulations were carried out by the software tool MicroWave Studio (MWS) based on the finite integration technique. This software has the great advantage of allowing the importation of the board configuration directly from CAD. It is known that full-wave simulation tools are suitable to provide a detailed analysis of complex structures, but they can require significant computational resources in terms of memory requirements and computing time. In order to achieve good results while keeping the computational requirements reasonable, the following crucial choices were made in setting up the simulation:

- The board layouts were imported by Gerber from the mechanical drawing of the board considering all the discontinuities such as vias, holes, and cuts for SMA connectors and for mounting decoupling capacitors.

- Power and ground planes were modeled as perfect electric conductors, i.e., no electric field tangential component $\left(E_{t}=0\right)$.

- The board side planes were modeled as perfect magnetic conductors, i.e., no magnetic field tangential component $H_{t}=0$ ). This choice is reasonable when the analysis investigates the resonance frequencies of the board, and allows a strong reduction of the computational domain.

- The board excitation through SMA connectors was modeled by using a discrete port of length $l=0.94 \mathrm{~mm}$ placed in the ground plane as shown in Fig. 6(a). For a more accurate modeling of the board excitation, the SMA connector should be taken into account into the model, leading to a bigger computational domain and longer simulation time. In any case, as will be shown later by comparing the simulations with measurements,

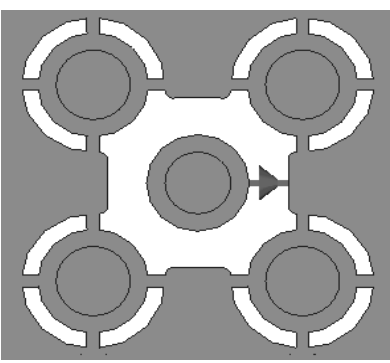

(a)

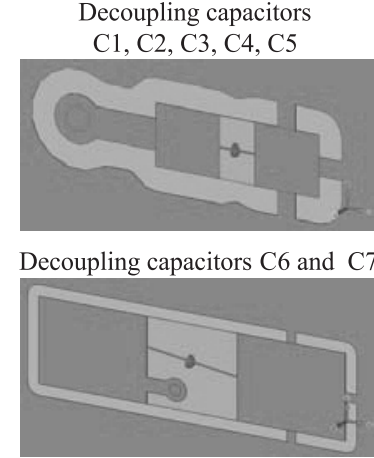

(b)
Fig. 6 MWS modeling of SMA connectors by a discrete port (a) and of decoupling capacitors by lumped elements (b).

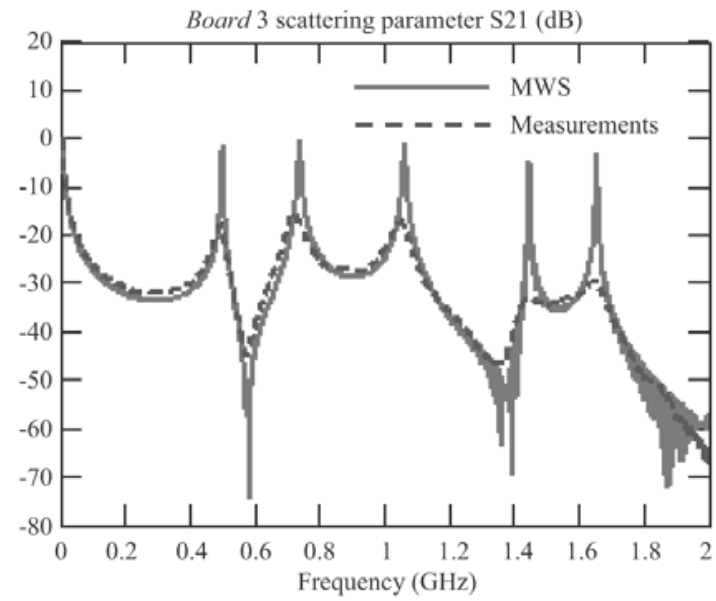

(a)

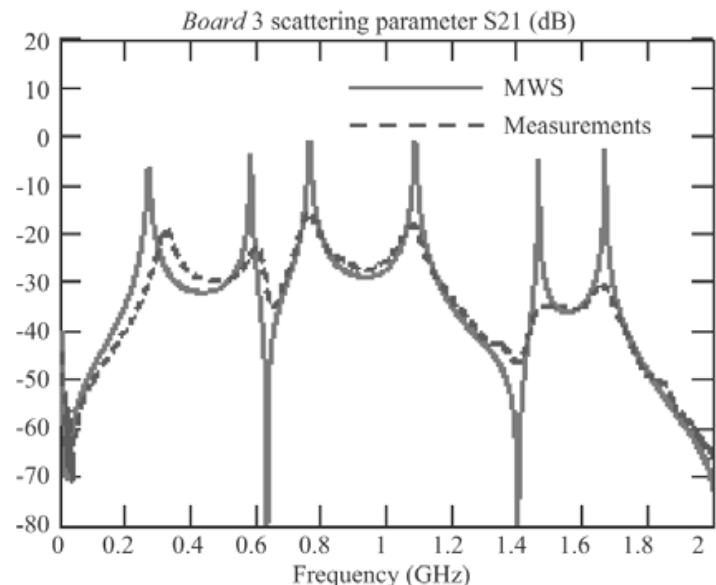

(b)

Fig. 7 Scattering parameter $S_{21}$ in case of Board 3: without (a) and with (b) decaps.

the approximation introduced will provide a good accuracy.

- Decoupling capacitors were modeled by lumped circuit elements having the nominal values of in Table 1. The lumped element length of capacitors C1-C5 was $0.6 \mathrm{~mm}$, while that of capacitors C6-C7 was $3.18 \mathrm{~mm}$. 


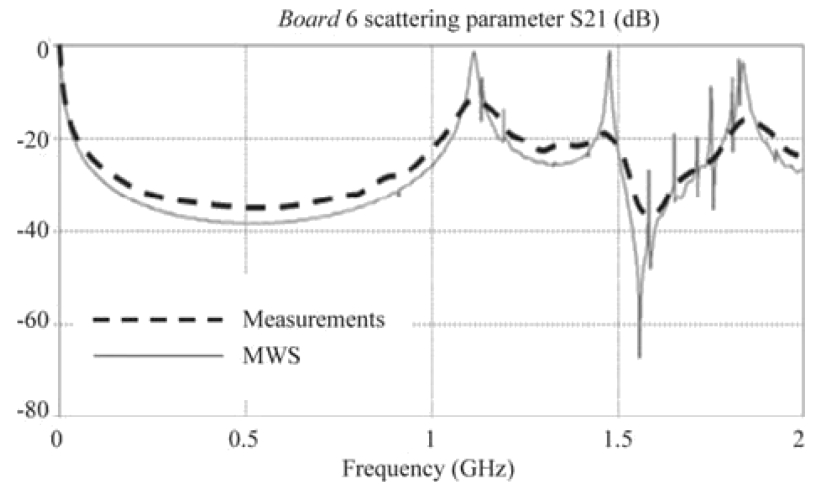

(a)

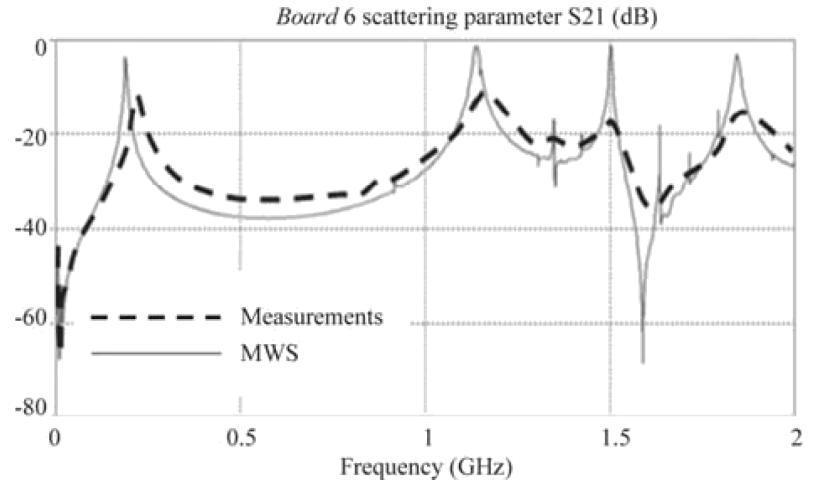

(b)

Fig. 8 Scattering parameter $S_{21}$ in case of Board 6: without (a) and with (b) decaps.

Results of MWS simulations obtained in the cases of Boards 3 and 6 without and with decaps are shown in Figs. 7, 8. Measurements and numerical simulations for both Boards 3 and 6 are in good agreement, apart from the sharper resonance peaks computed by MWS and a slight shift of the first resonance when decaps are present. The sharper peaks obtained by MWS are due to the fact that losses were neglected in the simulations in order to speed up the calculation. The reduction of this first resonance frequency computed by MWS is due to the extra inductance associated to the finite dimension of the lumped elements used to model the capacitors. Note that especially in case of C6 and C7, this inductance is not negligible, it is on the order of $2 \mathrm{nH}$ as can be seen by applying (4), and assuming $r_{\mathrm{via}}=0.1 \mathrm{~mm}$. In case of Board 6 there are also some discrepancies above $1.5 \mathrm{GHz}$ due to some spikes present in the numerical solutions. Simulations also confirm that decoupling capacitors are effective in lowering $S_{21}$ up to $150 \mathrm{MHz}$. An additional resonance peak appears at $200 \mathrm{MHz}$ due to the inductive effect of the capacitors. Moreover, above $300 \mathrm{MHz}$ the $S_{21}$ parameter is not affected by capacitors. To verify the impact of real test board cuts, holes, vias, and decoupling capacitor pads, an additional investigation was performed by MWS simulations. Two configurations of simplified Board 1 wihout and with vias, cuts, and holes are modeled (see Fig. 9(a)). The comparison of simulation results is shown in Fig. 9(b) and it clearly highlights the $S_{21}$ increase due to the presence of vias, cuts and holes. This section has shown that
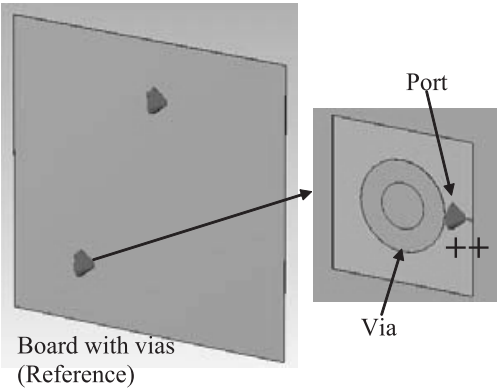

(a)

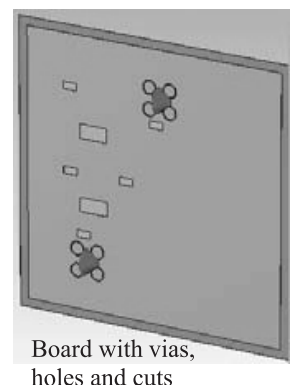

holes and cuts

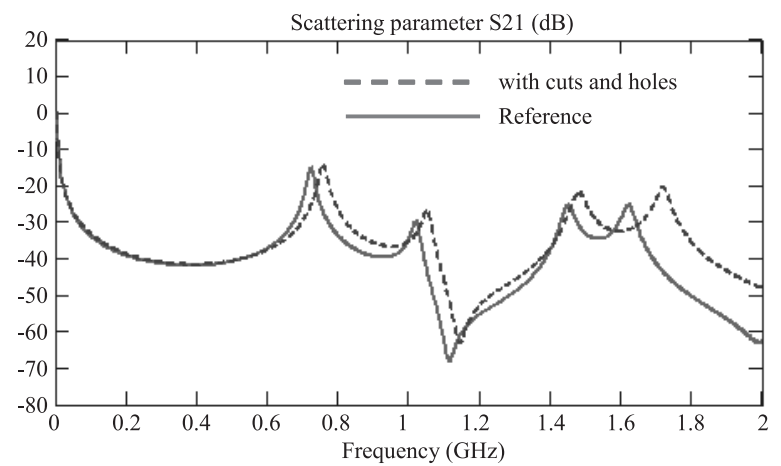

(b)

Fig. 9 MWS model of the simplified Board 1 without and with cuts and holes (a), and calculated scattering parameter $S_{21}$ (b).

the full-wave simulation can reproduce the measurements with very good accuracy even in the case of complex multilayer boards. The main drawback of this approach is the long computational time required, and it doesn't allow fast investigation of physical phenomena causing EMI. For this purpose, it is convenient to use other approaches such as the cavity model [2], [4] which is very suitable for modeling parallel solid planes with and without decoupling capacitors, or SPICE circuit modeling that allows a fast investigation of EMI phenomena involved [2].

\section{Low-Frequency SPICE Model for Decoupling Ca- pacitor Investigation}

When the interest is focused on the effects of decoupling capacitors only, a low-frequency (LF) SPICE circuit can be used as a very simple, convenient, and fast prediction tool [4]. However, the LF circuit model does not account for PCB resonances due to the cavity effect. The main parameters considered by this model are:

1. The intrinsic capacitance $C_{\mathrm{PCB}}$ between the two planes (i.e., inter-plane capacitance).

2. The inductance $L_{\mathrm{S}}$ associated to the source.

3. The equivalent series $\mathrm{R}, \mathrm{L}, \mathrm{C}$ circuit of the decoupling capacitors.

A critical issue in setting up this circuit is the calculation of the source inductance $L_{\mathrm{S}}$ that can be expressed as

$$
L_{\mathrm{S}}=L_{\text {board }}+L_{\mathrm{via}}
$$




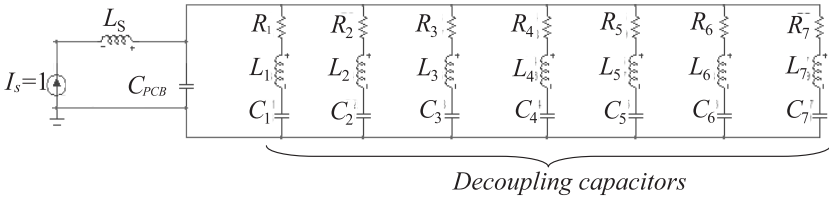

Fig. 10 LF-circuit model of Board 1 with decoupling capacitors having the nominal values of parasitic parameters as reported in Table 1.

where $L_{\text {board }}$ is the radial inductance of the board seen from the connection points of the source to the PCB, and $L_{\mathrm{via}}$ is the via inductance. Under the assumption that the field perturbation due to the switching device travels outwards from the component with cylindrical symmetry, the generated waves move as in a radial transmission line which behaves as a non-uniform TL with line inductance and capacitance depending on the radial distance $r$ from the origin [2], [13], [14]. In that case, the board radial inductance $L_{\text {board }}$ can be calculated by the radial transmission line theory as:

$$
L_{\text {board }}=\int_{r_{\text {via }}}^{r_{2}} \mu d /(2 \pi r) d r
$$

where $r_{\text {via }}$ is the source via radius, and $r_{2}$ defines the board maximum extension. Note that since the radial inductance decreases inversely as the distance from the source, it is reasonable to neglect the inductance at distances greater than twenty times rvia and assume $r_{2}=20 r_{\text {via }}$ in (3). The via inductance can be derived by the application of the partial inductance concept as:

$$
L_{\text {via }}=\frac{\mu_{0}}{2 \pi} d\left(\ln \left(2 d / r_{\text {via }}\right)-1\right)
$$

where $d$ is the substrate thickness. This expression is valid for $r_{\text {via }} \ll d$ [2]. When $r_{\text {via }}$ is comparable with $d$, a more complicated expression should be used [2]. In the case of Board $1, L_{\mathrm{via}}$ is negligible compared to $L_{\text {board }}$, as $d$ very small, and $L_{\mathrm{S}}=0.072 \mathrm{nH}$ is obtained. Note that this approach based on the radial TL theory allows a fast approximate estimation and an easy physical interpretation of the $L_{\mathrm{S}}$ parameter. A more accurate prediction of $L_{\mathrm{S}}$ can be derived by the cavity mode method as described in [15]. By this approach, in the case of Board $1 L_{\mathrm{S}}=0.095 \mathrm{nH}$ is obtained. The LF circuit model of Board 1 is shown in Fig. 10 where $L_{\mathrm{S}}=0.095 \mathrm{nH}, C_{\mathrm{PCB}}=3.173 \mathrm{nF}$, and the RLC circuits of decaps have the nominal values shown in Table 1. The PDN impedance calculated by the LF circuit in case of simplified Board 1 and Board 1 with decaps are shown in Fig. 11, and the results are compared with those obtained by the more accurate high-frequency circuit model described in [2]. Note that the first dip in case of simplified board (see Fig. 11(a)) is due to the inductance associated to the source. A filtered board would have no resonance points if decaps were not affected by parasitic inductance as shown by the dotted line in Fig. 11(b). In this case, even the first series resonance due to the source inductance disappears due to the fact that $C_{\mathrm{PCB}}$ is much lower than decaps capacitance. It can be also observed that the resonant frequencies exhibited by $Z_{\text {PDN }}$ in Fig. 11 coincides with those of scattering parameter

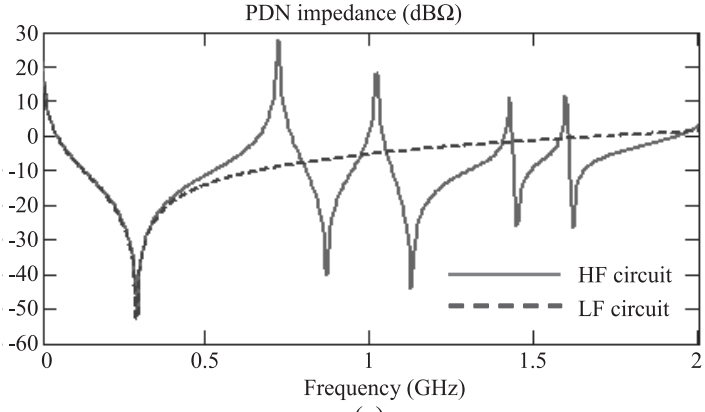

(a)

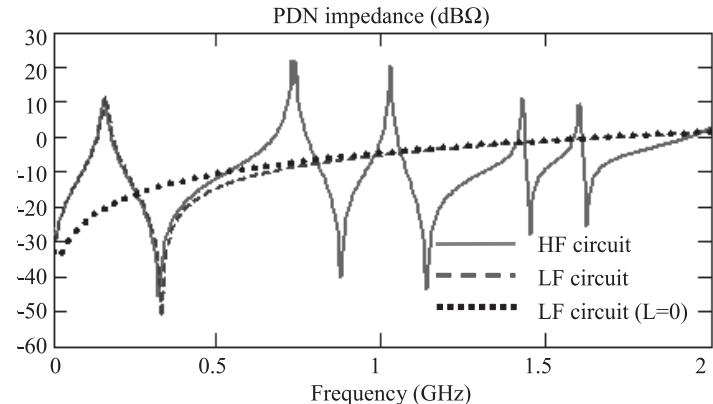

(b)

Fig. 11 Comparison $S_{21}$ calculated by the HF- and by the LF-circuit models: bare board 1 (a) and board 1 with decaps (b).

$S_{21}$ shown in Fig. 9. The LF circuit model is very simple to form and allows fast prediction, but it can compute the first resonant frequency only.

\section{Conclusions}

Boards of increasing complexity have been investigated by experimental measurements and by a full-wave prediction tool. The investigation has highlighted that:

- $Z_{\mathrm{PDN}}$ is the main parameter to show the ability of the fixes adopted (i.e., decoupling capacitors, embedded capacitance, etc.) in mitigating EMI phenomena in a PCB such as delta-I noise and emissions.

- Numerical tools are suitable to reproduce $S$ parameters obtained by measurements, and therefore $Z_{\mathrm{PDN}}$ also, for complex boards with several planes where vias, short cuts and power islands are present.

- A correct interpretation of numerical simulations and measurements can be done if the associated inductance to the sourcing port and the nearby discontinuities for SMA connector are taken into account.

- The effectiveness of decoupling capacitors is limited to the low frequency range (i.e., below $500 \mathrm{MHz}$ )

- The use of a full-wave analysis is required to predict resonant frequencies of complex boards with vias, cuts, splits, multiple planes.

\section{References}

[1] IEC 61967-2, Integrated circuits - Measurement of electromagnetic emissions, $150 \mathrm{kHz}$ to $1 \mathrm{GHz}$ - Part 2: Measurement of radiated emissions - TEM cell and wideband TEM cell method. 
[2] S. Caniggia and F. Maradei, Signal Integrity and Radiated Emission of High-Speed Digital Systems, John Wiley \& Sons, The Atrium, Southern Gate, Chichester, West Sussex, PO198SQ, UK, 2008.

[3] J. Knighten, B. Archambealt, J. Fan, G. Selli, and S. Connor, "PDN design strategy: I. Ceramic SMT decoupling capacitors - What value should I choose?," IEEE EMC Society Newsletter, vol.207, pp.34-41, 2005.

[4] T. Hubing, J. Drewniak, T. Van Doren, and D. Hockanson, "Power bus decoupling on multilayer printed circuit boards," IEEE Trans. Electromagn. Compat., vol.37, no.2, pp.155-167, May 1995.

[5] Z.L. Wang, O. Wada, Y. Toyota, and R. Koga, "Convergence acceleration and accuracy improvement in power bus impedance calculation with a fast algorithm using cavity modes," IEEE Trans. Electromagn. Compat., vol.47, no.1, pp.2-9, Feb. 2005.

[6] C. Wang, J. Mao, G. Selli, S. Luan, L. Zhang, J. Fan, D. Pommerenke, R. DuBroff, and J. Drewniak, "An efficient approach for power delivery network design with closed-form expressions for parasitic interconnect inductances," IEEE Trans. Adv. Packag., vol.29, no.2, pp.320-334, May 2006

[7] T.H. Hubing, J.L. Drewniak, T.P. Van Doren, and D. Hockanson, "Power bus decoupling on multilayer printed circuit boards," IEEE Trans. Electromagn. Compat., vol.37, no.2, pp.155-165, May 1995.

[8] J.H. Kim and M. Swaminathan, "Modeling of multilayer power distribution planes using transmission matrix method," IEEE Trans. Adv. Packag., vol.25, no.2, pp.189-199, May 2002.

[9] X. Chen, M. Cracraft, Y. Zhang, J. Zhang, J.L. Drewniak, B. Archambeault, and S. Connor, "An efficient implementation of parallel FDTD," IEEE Int. Symp. Electromagn. Compat., pp.1-5, Honolulu, July 2007.

[10] B. Archambeault and A.E. Ruelhi, "Analysis of power/ground plane EMI decoupling performance using the partial-element equivalentcircuit method," IEEE Trans. Electromagn. Compat., vol.43, no.4, pp.437-445, Nov. 2001.

[11] X.-C. Wei, E.-P. Li, E.-X. Liu, and X. Cui, "Efficient modeling of rerouted return currents in multilayered power-ground planes by using integral equations," IEEE Trans. Electromagn. Compat., vol.50, no.3, pp.740-743, Aug. 2008.

[12] S. Hall, G. Hall, and J. McCall, High-Speed Digital System Design - A Handbook of Interconnect Theory and Design Practice, John Wiley \& Sons, New York, NY, 2000.

[13] S. Caniggia, V. Costa, and R. Preatoni, "Investigation of EMI on multilayer circuit boards: DeltaI-noise and power supply decoupling," IEEE 1996 International Symp. on Electromagn. Compat., pp.436-441, Santa Clara, Aug. 1996.

[14] S. Caniggia, V. Costa, and L. Vitucci, "Investigation of EMI on multilayer circuit boards: Radiated emission,” IEEE 1996 International Symp. on Electromag. Compat., pp.316-321, Santa Clara, Aug. 1996.

[15] L. Ren, J. Kim, G. Feng, B. Archambeault, J. Knighten, J. Drewniak, and J. Fan, "Frequency-dependent via inductance for accurate power distribution network modeling," IEEE 2009 Int. Symp. on Electromag. Compat., pp.63-68, Austin, Aug. 2009.

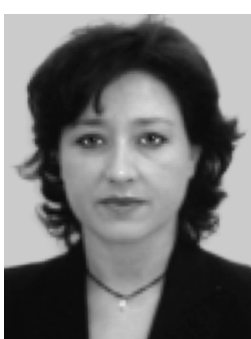

Francescaromana Maradei (M'96, S'06) received the Laurea degree in electrical engineering (cum laude) from Sapienza University of Rome in 1992, the Diplome d'Etudes Approfondies (DEA) in electrical engineering from the Institut National Polytecnique de Grenoble, Laboratoire d'Electrotechnique de Grenoble, France, in 1993, and the Ph.D. degree in Electrical Engineering from the Sapienza University of Rome "La Sapienza," Italy, in 1997. She joined the Department of Electrical Engineering at the the Sapienza University of Rome "La Sapienza" in 1996 where she is currently associate professor. Her main interests are in numerical techniques and their application to EMC problems (shielding and transmission line analysis). She is author of more than 100 papers and coauthor of the book "Signal Integrity and Radiated Emission of High-Speed Digital Systems," published by John Wiley \& Sons Ltd in November 2008. She received the James Melcher Price Paper Award with the paper "Analysis of upset and failures due to ESD by the FDTD-INBCs method," IEEE Trans. Industry Applications, vol.38, no.4, July/Aug. 2002, pp.1009-1017; the Oral Presentation Best Paper Award at the International Symposium on Electromagnetic Compatibility - EMC ROMA 1994, Rome, Italy, and the Poster Presentation Best Paper Award at the International Symposium on Electromagnetic Compatibility —EMC EUROPE 2000, Brugge, Belgium. Prof. Maradei is member of the Board of Directors of the IEEE Electromagnetic Compatibility Society since 2005 , where she is also serving as 2010-2011 President. She has served as Chapter Coordinator (2005-2009) and as an Associate Editor of the IEEE Transactions on EMC (1999-2000). Since 1998 she is member of the Editorial Board of IEEE Conference on Electromagnetic Field Computation (CEFC) and of COMPUMAG Conference.

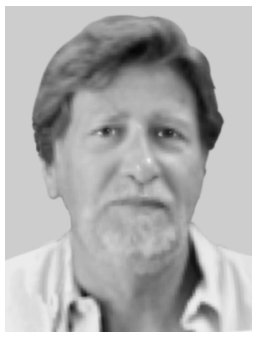

Spartaco Caniggia (M'97) was born in Venice, Italy, in 1948. He received the Laurea degree in electronic engineering from the University of Padua, Padua, Italy, in 1972. In 1975, after military service, he joined Siemens/Italtel, Milan, Italy, where he retired as a Physical Design and Electromagnetic Interference/Electromagnetic Compatibility (EMI/EMC) Manager for the Business Unit Products in 2005. He was a part-time Lecturer with the University of Rome "La Sapienza" L'Aquila, Milan, and Turin for signal integrity and EMC activities. From 2002 to 2004, he was with the EMC consortium of the University of Missouri-Rolla, Rolla. He is currently an EMC consultant. His research interests included the development of computer-aided design tools for SI and EMC; mathematical models for electrostatic discharge, shielding, grounding and wiring; methods for designing and testing information technology equipment to comply with EMC standards. He is the author of about 60 technical papers published in several journals and conference proceedings. Dr. Caniggia received the Oral Presentation First Best Paper Award at the National EMC Symposium in 1988 and the Oral Presentation Second Best Paper Award at the IEEE International Symposium on Electromagnetic Compatibility in 1996. He is a member of working group SC77B MT12 of the International Electrotechnical Commission (IEC) for the immunity of the apparatus to transient interferences such as ESD, EFT, Surge, etc. He is the co-author of the "Signal Integrity and Radiated Emission of High-Speed Digital Systems" book, published by John Wiley \& Sons Ltd in November 2008. 


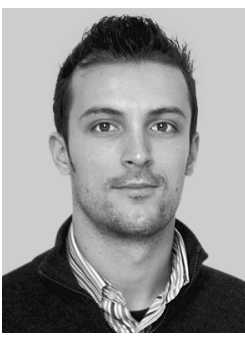

Nicola Inverardi was born in 1981, in Brescia. He received the Laurea degree in Telecommunication Engineering from Universit degli Studi of Brescia in 2006. He joined Euro Instruments in 2006 working for both the R\&D and CAD department. He is currently involved in PCBs EMC Signal and Power Integrity simulations with 3D and 2D numerical tools. His areas of interest include electrical schematics design, SW programming for IC testing systems, semiconductors reliability trials and EMC/EMI tests.

He is currently working with the R\&D department.

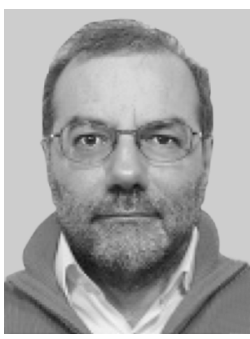

Mario Rotigni was born in Bergamo, Italy, 1958 and received a diploma in Electrical Engineering in 1977. He joined the R\&D Department of Magrini Galileo in 1978 were he took part of the development of instrumentation and systems to test in Laboratory and production lines, as well as control in field, high-voltage circuit breakers and their auxiliary equipments. The equipments had to operate in very hostile Electromagnetic Environment. He joined $\mathrm{Au}-$ diotel in 1990 to develop an ATE dedicated to test in productions the microcontrollers integrated circuits. Since 1993 he is with STMicroelectronics, holding various position in the Engineering, Design and Validation of Microcontrollers and Embedded FLASH Memories. He is currently with the Automotive Product Group taking care of the EMC Evaluation of the Integrated Circuits and Board Hardware Design. His main interests are in the Embedded Applications, Low Emission/Low Susceptibility application design and EMI/EMS at component level. 